\title{
Reconstruction of a Large Full-Thickness Alar Defect Using an Extended Free Composite Flap from the Pinna: A Case Report
}

\author{
Apostolos Vlachogiorgos, MD, MRCS ${ }^{1}$ Titus-Andrei Grecu, MD ${ }^{1} \quad$ Andrej Salibi, MD, MRCS, MSc ${ }^{1}$ \\ Deemesh Oudit, MB, BS, FRCSEd(Plast) $)^{1,2}$ \\ ${ }^{1}$ Department of Plastic Surgery, The Christie Hospital, Manchester, \\ Address for correspondence Apostolos Vlachogiorgos, MD, MRCS, \\ United Kingdom \\ 2 Institute for Cancer Sciences, Faculty of Biology, University of \\ Manchester, Manchester, United Kingdom \\ Department of Plastic Surgery, The Christie Hospital, 550 Wilmslow \\ Road, Manchester M20 4BX, United Kingdom \\ (e-mail: apostolos.vlachogiorgos@christie.nhs.uk).
}

J Reconstr Microsurg Open 2018;3:e78-e81.

\begin{abstract}
Alar reconstruction can pose a challenging task in reconstructive surgery. Herein, we describe a case of a large full-thickness alar defect (involving the full- thickness of the left ala, $50 \%$ of the tip of the nose and extending over the left nasal sidewall and cheek) that was reconstructed using a contralateral free composite pinna flap, which extended into the right temple. A 70-year-old man with a squamous cell carcinoma to the left ala underwent surgical excision and immediate reconstruction with an extended contralateral free composite pinna flap based on a branch of the right superficial temporal artery supplying the helical root and the skin paddle of the supra-auricular area. The patient had an uneventful recovery and the result was aesthetically pleasing without compromising the nostril or the external nasal valve. Based on this case, a free

\section{Keywords}

- nasal ala

- alar reconstruction

- nasal reconstruction composite flap incorporating the contralateral root of helix and adjacent tissue from the temporal region is an option that could be used in a single-staged procedure for reconstruction of large full-thickness alar defects. One of the challenges of performing free flaps in this area is the paucity of suitable recipient veins. This can be reliably addressed with a vein graft.
\end{abstract}

Alar reconstruction can pose a challenging task in reconstructive surgery. A large variety of local flaps have been described in the past for reconstruction of relatively small alar defects. For the reconstruction of full-thickness defects of the ala, in addition to composite grafts, there are more complex local, regional, or pedicled flap options. ${ }^{1,2}$ However, the best option for reconstruction of full-thickness alar defects is a free vascularized composite tissue from the ear, as first described in 1985 by Parkhouse and Evans ${ }^{3}$ and others. ${ }^{4}$ In 1993, nasal reconstruction with free auricular flaps became more popular with the work of Pribaz and Falco. ${ }^{5}$ Since then, several authors have further described free composite flaps from the ear, not only for alar ${ }^{6,7}$ but also for columellar, ${ }^{8}$ lip, and intraoral reconstruction. ${ }^{9,10}$

received

February 13, 2018 accepted after revision April 28, 2018
DOI https://doi.org/

10.1055/s-0038-1675409. ISSN $2377-0813$.
In this report, we describe a case of reconstruction of a large full-thickness alar defect (involving the full thickness of the left ala, $50 \%$ of the tip of the nose and extending over the left nasal sidewall and cheek), using a free extended composite flap from the pinna incorporating a relatively large segment of tissue from the temporal region to reconstruct the adjacent skin defect of the cheek.

\section{Case Report}

A 70-year-old Caucasian man was referred to our clinic with a rapidly growing lesion on his left ala (-Fig. 1). An initial punch biopsy proved that the lesion was a well-differentiated squamous cell carcinoma with no lymphovascular or

Copyright $\odot 2018$ by Thieme Medical Publishers, Inc., 333 Seventh Avenue, New York, NY 10001, USA. Tel: +1(212) 584-4662.

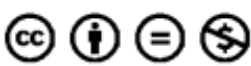

License terms 

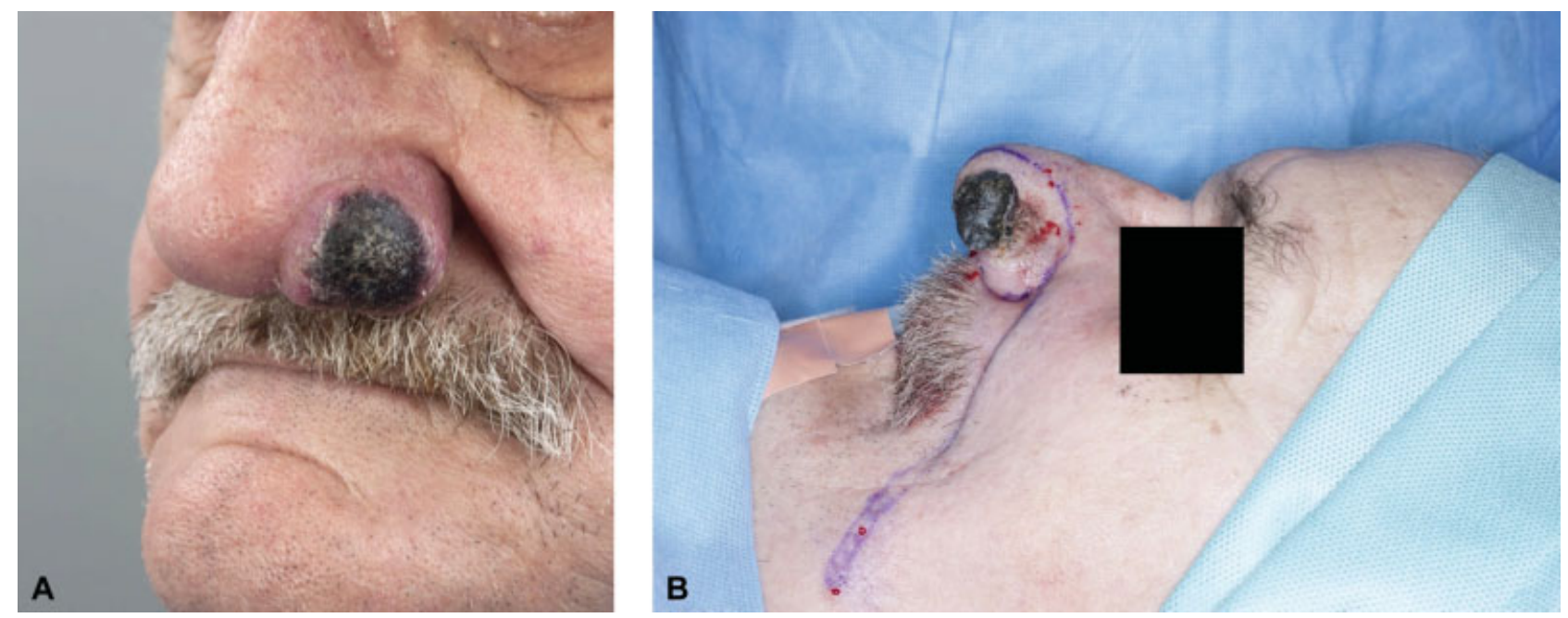

Fig. 1 A 70-year-old Caucasian man with a rapidly growing squamous cell carcinoma to his left ala. (A) Lateral view of the lesion at the outpatient clinic. (B) Lateral view of the lesion on table.

perineural invasion and no clinical evidence of nodal metastases.

Following excision of the lesion and after confirming margin clearance with frozen sections (-Fig. 2), a paper template was used to map out the defect and configure and mark the tissue required for reconstruction, trying as best as possible, to match like with like. The contralateral ear was used as a donor site as the shape of the superior helical rim mirrors the contour of the nasal ala. The donor site was then marked according to the designed template ( - Fig. 3 ). Due to the large size of the defect and the fact that it included multiple aesthetic subunits, the flap was extended to include a vascularized skin paddle from the supra-auricular area to provide coverage to the dorsum of the nose, the left nasal sidewall, and the left cheek adjacent to the alar groove.

The right superficial temporal artery was identified via a longitudinal preauricular incision starting from the inferior point of the flap toward the angle of the mandible. The superficial temporal vessels were identified and dissected. On further dissection in a cephalad direction, a branch of the

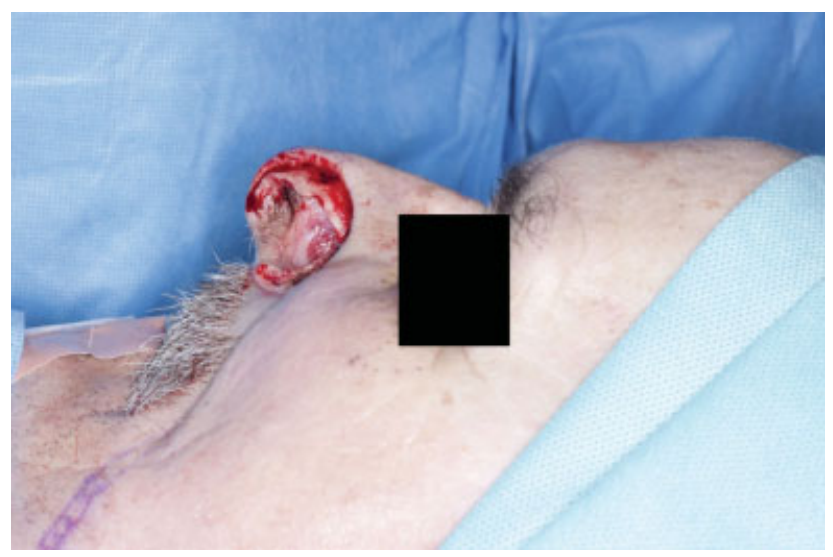

Fig. 2 Large alar defect after the excision of the lesion. The defect involves the full thickness of the left ala, $50 \%$ of the tip of the nose and extends over the left nasal sidewall and cheek. superficial temporal vessel was noted heading to the superior pole of the pinna. This branch was carefully preserved and incorporated into the flap. The adjacent skin paddle was elevated in a subfascial plane to preserve the vasculature of the flap. This extended composite flap based on the branches of the superficial temporal vessels incorporated the upper third of the helix as well as a vascularized segment of tissue from the supra-auricular temporal region.

The helical fold of the ear was advanced and sutured directly to the root of the helix maintaining a supra-auricular groove, which is important when wearing spectacles. The adjacent temporal defect was resurfaced with a full-thickness skin graft.

The terminal branch of the facial artery was easily identified and dissected in the nasolabial groove and was anastomosed to the superficial temporal artery. However, despite extensive meticulous dissection, no suitable recipient veins were identified in the perinasal or cheek regions. As a result, the superficial temporal vein was anastomosed to a branch of the facial vein in the region of the submandibular gland via a

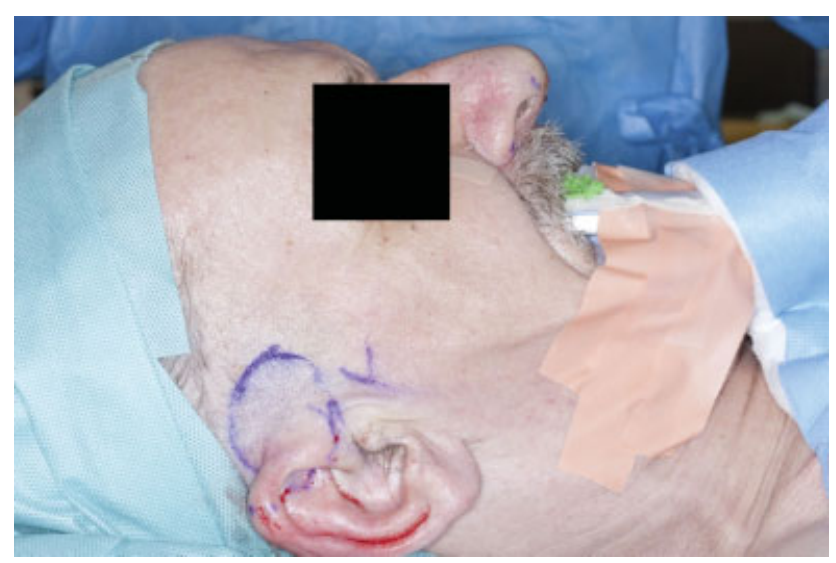

Fig. 3 Flap marking according to the designed template. 
vain graft. The vein graft was harvested from the distal forearm and resulted in an additional scar. All the anastomoses were performed in end-to-end fashion.

The flap was finally inset into the defect with the helical rim reconstructing the left nasal alar margin, and the vascularized supra-auricular skin paddle was used to reconstruct the resurfacing the remainder of the dorsum of the nose, nasal sidewall, and adjacent cheek.

The patient had uneventful recovery and was discharged 5 days postoperatively. He was reviewed in the outpatient clinic 6 months postoperatively with a satisfactory aesthetic outcome ( - Fig. 4). The nostril maintained an almost similar size and shape to the contralateral side. The alar groove is still slightly bulky, but it is not a concern for the patient. Functionally, he has no difficulty in breathing through the left reconstructed nostril and the external nasal valve is not compromised.

\section{Discussion}

Reconstruction of a full-thickness alar defect is one of the most challenging procedures in plastic surgery and utilizes the various principles of facial and nasal reconstruction. ${ }^{11}$ Traditionally, this requires multiple serial procedures to obtain a near-aesthetically pleasing and functional outcome. These procedures often tend to leave a bulky result, ${ }^{5}$ which is difficult to thin and erase the alar groove resulting in a negative aesthetic outcome.

The use of a composite graft is usually a good option for single-staged reconstruction. It does not involve microsurgery; less time and less skill are needed. However, composite grafts can only be used for relatively small defects (less than $2 \mathrm{~cm}$ ). ${ }^{4}$ Furthermore, resorption of the cartilage can lead to notching with a suboptimal and an overall unpredictable result.

Parkhouse and Evans ${ }^{3}$ described a free composite flap in 1985 for reconstruction of full-thickness alar defects but stated that it is performed with difficulty and hence with unreliable results. Despite reports of readily accessible suitable veins for microsurgical anastomosis in the perinasal area, ${ }^{9,10,12}$ there is a general paucity of recipient veins around this region and this contributes to the difficulty of this reconstructive option. This undoubtedly has resulted in the poor popularity of free tissue transfer as an option for the reconstruction of these defects.

There have been several reports in the literature highlighting the paucity of suitable regional recipient veins. Zhang et $\mathrm{al}^{13}$ presented 63 cases of nasal reconstruction with free helical flaps. In $70 \%$ of their cases, they chose to use vein grafts due to nonsuitable veins. Li et al ${ }^{14}$ published their experience of four free auricular flaps, where vein grafts were used in all four cases due to the lack of recipient veins in the nasolabial area. The paucity of good recipient veins can make free tissue reconstruction difficult and lead to venous congestion of the flap. Whenever recipient veins cannot be used, this can be addressed reliably with a vein graft to the submandibular branch of the facial vein in the neck. ${ }^{5,13,14}$ This additional step of the procedure leaves a scar on the forearm or other donor but does not dramatically increase the operating time. The benefit of a vein graft in this case far outweighs the additional time for the procedure and the donor-site scar, as it significantly reduces the risk of venous congestion and improves flap survival.

We believe that the optimal option for the reconstruction of full-thickness alar defects is a free composite pinna flap. We have shown that a large alar defect involving multiple anatomical subunits can be reconstructed by harvesting the contralateral helical rim along with an adjacent skin paddle supplied by the same branch of the superficial temporal artery, offering an optimal reconstructive result. There are various advantages behind this option. First, an extended and pliable flap can be harvested in a single-staged procedure. Second, raising the flap is straight forward due to the constant and reliable anatomy of the superficial temporal artery. ${ }^{10,13}$ Finally, we believe that the helical rim is an ideal donor site as it can reform the alar shape but also has similar color and texture resulting in a good aesthetic and functional outcome.

In summary, an extended free composite flap incorporating the contralateral root of helix and adjacent tissue may be used in a single-staged procedure for reconstruction of a fullthickness alar defect involving multiple adjacent aesthetic
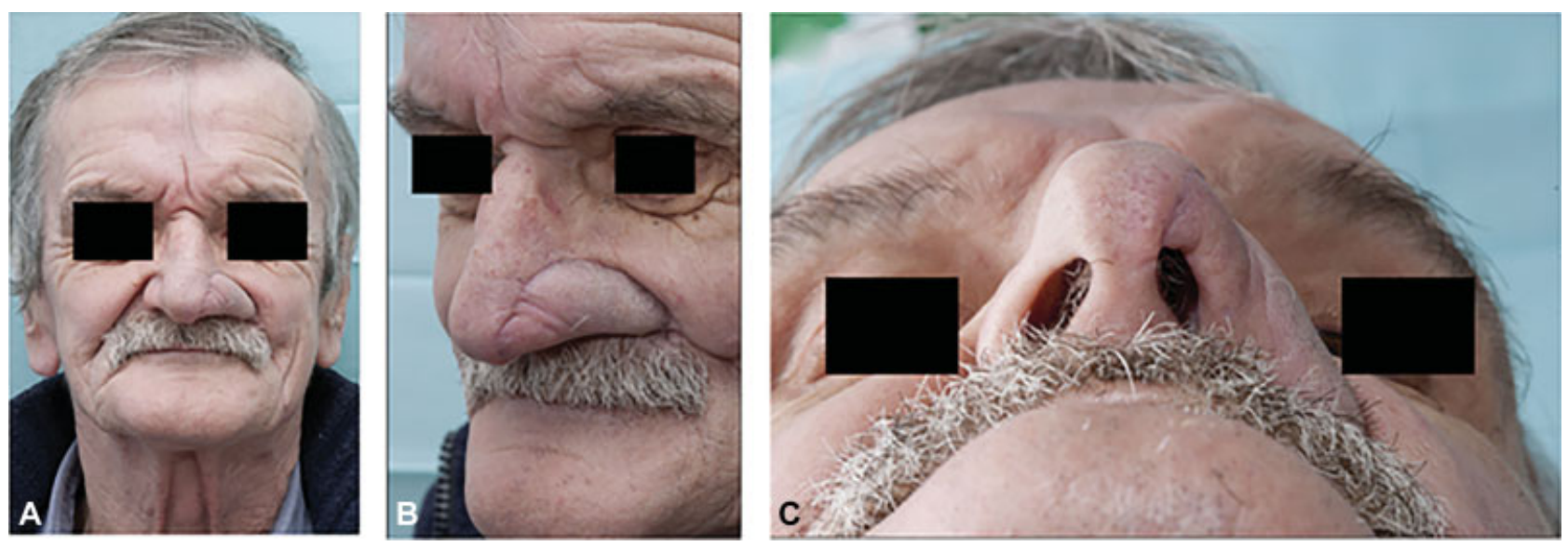

Fig. 4 Six months postoperatively. (A) Front view. (B) Left lateral view. (C) Inferior view. The nostril maintained an almost similar size and shape to the contralateral side. 
subunits of the face. This has also highlighted the versatility of the free composite flap of the helical root based on the superficial temporal artery. There is a general paucity of recipient veins in this area. In cases when veins are not available, the use of a vein graft can reliably improve the outcome of the procedure by overcoming the risk of venous congestion with subsequent flap failure. We would recommend this free flap as the gold standard option for the reconstruction of full-thickness alar defects in suitable patients.

\section{Conflict of Interest \\ None declared.}

\section{References}

1 Driscoll BP, Baker SR. Reconstruction of nasal alar defects. Arch Facial Plast Surg 2001;3(02):91-99

2 Bakhach J, Riahi R, Demiri E, Conde A, Baudet J. The reverse auricular flap. A new flap [in Spanish]. Ann Chir Plast Esthet 1999; 44(03):253-261

3 Parkhouse N, Evans D. Reconstruction of the ala of the nose using a composite free flap from the pinna. Br J Plast Surg 1985;38(03): 306-313

4 Shenaq SM, Dinh TA, Spira M. Nasal alar reconstruction with an ear helix free flap. J Reconstr Microsurg 1989;5(01):63-67
5 Pribaz JJ, Falco N. Nasal reconstruction with auricular microvascular transplant. Ann Plast Surg 1993;31(04):289-297

6 Ozek C, Gurler T, Uckan A, Bilkay U. Reconstruction of the distal third of the nose with composite ear-helix free flap. Ann Plast Surg 2007;58(01):74-77

7 Tanaka Y, Tajima S, Tsujiguchi K, Fukae E, Ohmiya Y. Microvascular reconstruction of nose and ear defects using composite auricular free flaps. Ann Plast Surg 1993;31(04):298-302

8 Ozek C, Gundogan H, Bilkay U, Alper M, Cagdas A. Nasal columella reconstruction with a composite free flap from the root of auricular helix. Microsurgery 2002;22(02):53-56

9 Lassus P, Lindford AJ. Free temporal artery posterior auricular skin (TAPAS) flap: a new option in facial and intra-oral reconstruction. Microsurgery 2017;37(06):525-530

10 Lassus P, Husso A, Vuola J, Lindford AJ. More than just the helix: a series of free flaps from the ear. Microsurgery 2017

11 Menick FJ. Principles and planning in nasal and facial reconstruction: making a normal face. Plast Reconstr Surg 2016;137(06): 1033e-1047e

12 Shimizu F, Oatari M, Uehara M. Choice of recipient vessels for nasal ala reconstruction using a free auricular flap. J Plast Reconstr Aesthet Surg 2015;68(07):907-913

13 Zhang YX, Yang J, Wang D, et al. Extended applications of vascularized preauricular and helical rim flaps in reconstruction of nasal defects. Plast Reconstr Surg 2008;121(05):1589-1597

14 Li S, Cao W, Cheng K, et al. Microvascular reconstruction of nasal ala using a reversed superficial temporal artery auricular flap. J Plast Reconstr Aesthet Surg 2006;59(12):1300-1304 